\title{
Generation of Self Organized Critical Connectivity Network Map (SOCCNM) of Randomly Situated Water Bodies During Flooding Process
}

\author{
B. S. DAYA SAGAR* \\ Centre for Remote Sensing \& Information Systems, Department of Geoengineering, \\ Andhra University, Visakhapatnam-530 003, India
}

(Received in final form 13 April 2000)

\begin{abstract}
This letter presents a brief framework based on nonlinear morphological transformations to generate a self organized critical connectivity network map (SOCCNM) in 2-dimensional space. This simple and elegant framework is implemented on a section that contains a few simulated water bodies to generate SOCCNM. This is based on a postulate that the randomly situated surface water bodies of various sizes and shapes self organize during flooding process.
\end{abstract}

Keywords: Water bodies; Mathematical morphology; SOCCNM; Nonlinear transformations

\section{INTRODUCTION}

During flooding process the surface water bodies tend to self organize. This study that relates to know how the surface water bodies contact each other on a 2-dimensional space during flooding process is dealt with. Floods of different intensities generate typical surface water body organization network. The intuitive argument is that the smaller water bodies are more homogeneously distributed over a landmass than that of larger water bodies. A case study using real world data is tested in our earlier study that supports that this argument is justified [1]. Small water bodies will tend to merge each other with flood of small intensity, and the high degree of flood intensity makes the larger

\footnotetext{
* Present Address: Centre for Remote Imaging Sensing Processing (CRISP), Faculty of Science, The National University of Singapore, Lower Kent Ridge Road, Singapore 119260. e-mail: crsbsds@nus.edu.sg, bsdsagar@hotmail.com
} 
water bodies contact together. This is the postulate. For instance, how two closely situated surface water bodies self organize with small intensity of flooding can be visualized in spatial domain. Similarly randomly situated surface water bodies of several sizes and shapes will be self organized due to flooding. The critical map in 2-dimensional space of such self organization process of water bodies under the influence of flooding is simulated by following nonlinear mathematical morphological transformations. Such a map is visualized in terms of one pixel wide caricature. The randomness in the spatial distribution of surface water bodies decides the spatial structure of self organized critical connectivity network map. In a way it depicts the extinguishing points of self organized water bodies at critical state. The critical state is defined by the degree of flooding intensity. This map is termed in the present study as a self organized critical connectivity network map (SOCCNM).

In brief the second section enables the simple framework based on mathematical morphological transformations [2]. In third section, that follows, includes a sample study that consists of randomly distributed simulated water bodies. In what follows includes the mathematical framework.

\section{MATHEMATICAL MORPHOLOGICAL FRAMEWORK TO EXTRACT SOCCNM}

Henceforth, the section containing surface water body data is referred to as $\mathbf{M}$ that is a discrete binary level image consists of water bodies and no water body region. Dilation, erosion, opening and closing [2] are simplest quantitative morphological set transformations. The discrete binary level image, $\mathbf{M}$, is defined as a finite subset of Euclidean two dimensional space, $1 \mathrm{R}^{2}$ that can admit values 1 (water body or set) and 0 (no-water body region or set complement). Surface water bodies hereafter refer to as $M$ testify the presence of zones of supremums and infemums. Prior to understand the framework from geometrical point of view morphological dilations and erosions are defined as set transformations that expand and contract a set. The morphological operators can be visualized as working with two images. The image being processed is referred to as the set or set complement, and other image being a structuring template. Each structuring template has a designed shape that can be thought of as a probe of the image. The image can be decomposed by probing it with various structuring templates. The four basic morphological transformations dilation, erosion, and cascade of erosion-dilation and closing are defined with respect to the structuring element $S$ with scaling factor $e$, image $M$ and point $\mathrm{A}_{0} \in 1 \mathrm{R}^{2}$.

Dilation:

$$
\begin{gathered}
\delta_{\mathrm{s}}^{e}(\mathrm{M})\left(\mathrm{A}_{0}\right)=\mathrm{MAX}_{\mathrm{A} \in \mathrm{A}_{0}+\mathrm{e} . \mathrm{S}\left(\mathrm{A}_{0}\right)}(\mathrm{M}(\mathrm{A})) \\
\mathrm{e}=1,2, \ldots, \mathrm{n}
\end{gathered}
$$

Erosion:

$$
\epsilon_{\mathrm{s}}^{e}(\mathrm{M})\left(\mathrm{A}_{0}\right)=\mathrm{MIN}_{\mathrm{A} \in \mathrm{A}_{0}+\mathrm{e} . \mathrm{S}\left(\mathrm{A}_{0}\right)}(\mathrm{M}(\mathrm{A}))
$$

By duality, the opening of $\mathrm{M}$ by $\mathrm{S}$ comes from erosion first and then dilation. Thus the opening is defined as

Opening:

$$
\gamma_{\mathrm{s}}^{e}(\mathbf{M})=\delta_{\mathrm{s}}^{e}\left(\epsilon_{\mathrm{s}}^{e}(\mathrm{M})\right)
$$

The dilation followed by erosion is called closing transformation. These transformations can be carried out according to the multiscale approach [3]. In the multiscale approach, the size of the structuring template will be increased from iteration to iteration. Due to the noninvertible property of the superficially simple erosion and opening transformations and a logical operation SOCCNM can be generated.

These transformations are systematically used to remove the supremums " $\Lambda$ " in the set complement 
$\left(\mathrm{M}^{\mathrm{c}}\right)$. These topographically significant features enable the structural composition of the SOCCNM. This structural composition is sensitive to changes that occur due to both exogenic and endogenic processes. This self organized critical connectivity network map acts as a barrier that obstructs the further diffusion of water bodies, which are treated as source points from which rainfall diffuses and propagates towards the SOCCNM, during flooding. This propagation is of anisotropic in nature. The framework thus developed in this section is intended to isolate SOCCNM on a section, containing simulated water bodies, of size $200 \times 200$ pixels. In what follows in this section includes mathematical representation of sequential steps involved.

SOCCNM subsets of $n$th order: In the process of extraction of SOCCNM from the image, the opened version of each eroded version of $\mathrm{M}^{\mathrm{c}}$ needs to be subtracted from the corresponding eroded version. This process is mathematically shown as follows:

$$
\operatorname{SOCCNM}_{e}\left(\mathbf{M}^{\mathrm{c}}\right)=\left[\epsilon_{\mathrm{s}}^{e}\left(\mathbf{M}^{\mathrm{c}}\right)\right] /\left\{\gamma_{\mathrm{s}}\left[\epsilon_{\mathrm{s}}^{e}\left(\mathbf{M}^{\mathrm{c}}\right)\right]\right\}
$$

Once, several orders of subsets of SOCCNM are isolated from the image, they need to be made union by following the logical AND operation. The mathematical representation of this process to see the SOCCNM of M is as follows:

$$
\operatorname{SOCCNM}(\mathbf{M})=\bigcup_{\mathrm{e}=1}^{n}\left[\operatorname{SOCCNM}_{e}\left(\mathbf{M}^{\mathrm{c}}\right)\right]
$$

The above transformation needs to be performed to generate the SOCCNM.

\section{A SAMPLE STUDY}

As a sample study, a section that contains a few simulated water bodies (M) (Fig. 1a) has been used. This study is emphasized on the methodology and further scope to develop quantitative methods. The SOCCNM (Fig. 1b) is generated from the $\mathrm{M}$. The higher the spatial resolution the higher is the intricacy of the SOCCNM, vice versa. By following the mathematical framework thus developed on the data SOCCNM has been generated and shown in the Figure $1 \mathrm{~b}$.

Such an analysis can be acquired out on multidate data of a region (e.g., landform, river networks etc.) to understand the complex limnological processes. From the present study it is deduced that even minor degree of deformation that may be reflected on the SOCCNM structural composition can be extracted by following the procedure thus developed and tested. Moreover, spatiotemporal distribution of various geologically and
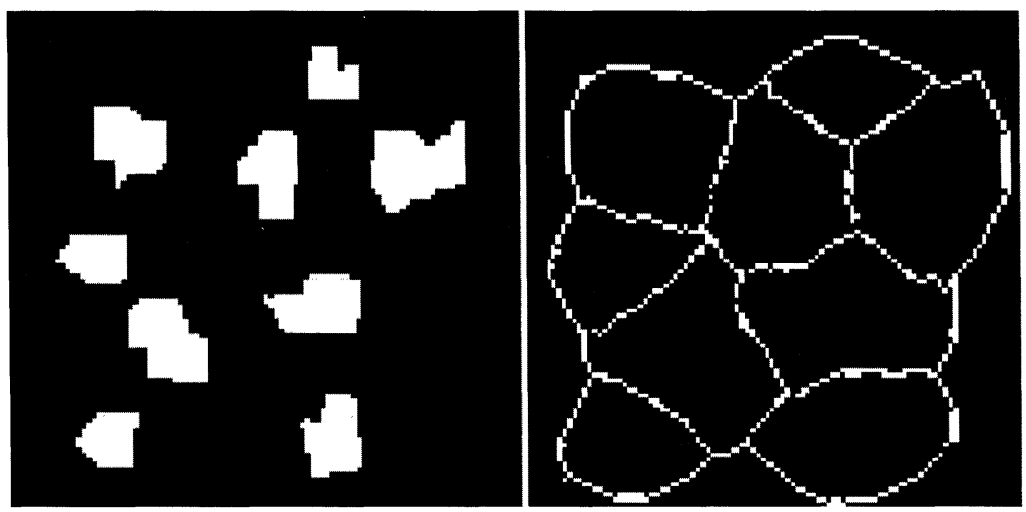

FIGURE 1 (a) A few simulated water bodies (M), and (b) the SOCCNM of the M. 
geomorphologically complex features can be studied further to develop cogent models of interest to geophysicists. Present study is an attempt to test the developed mathematical framework to extract SOCCNM from the section of simulated water bodies. However, these network maps across time intervals enable the spatio-temporal distribution that has not been covered in the present letter.

\section{References}

[1] Sagar, B. S. D., Is the spatial distribution of larger water bodies heterogeneous? (in press), International Journal of Remote Sensing.

[2] Serra, J. (1982). Image Analysis and Mathematical Morphology, Academic Press, London, p. 610.

[3] Maragos, P. A. and Schafer, R. W. (1986). Morphological skeleton representation and coding of binary images, I.E.E.E. Transactions on Acoustics, Speech, and Signal Processing, ASSP-34, 5, 1228-1244. 


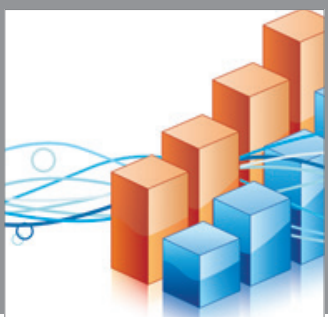

Advances in

Operations Research

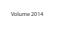

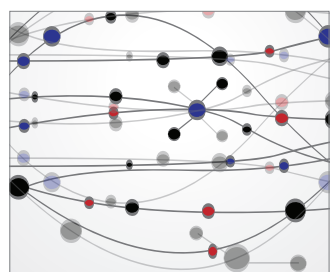

\section{The Scientific} World Journal
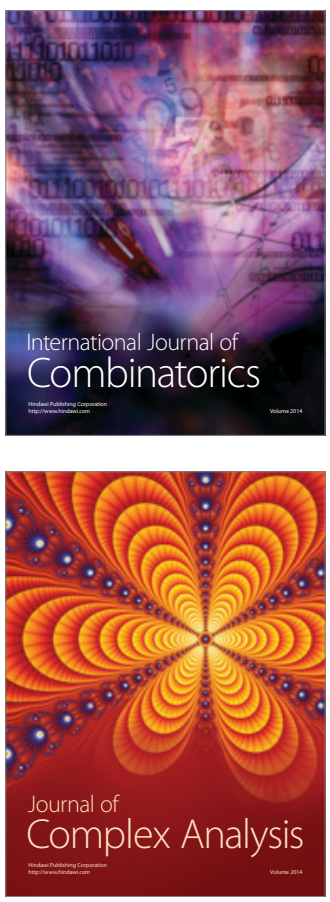

International Journal of

Mathematics and

Mathematical

Sciences
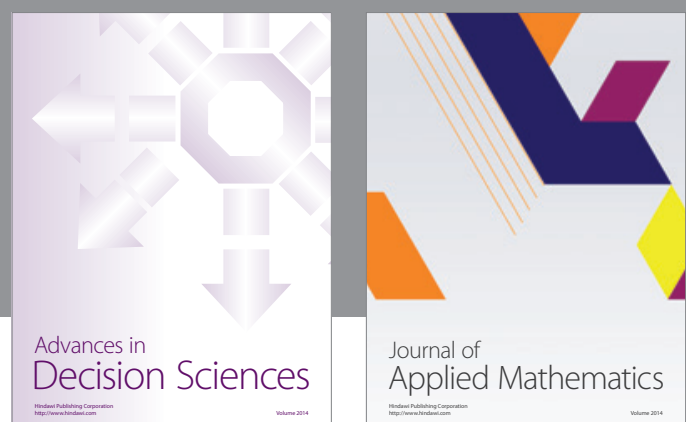

Journal of

Applied Mathematics
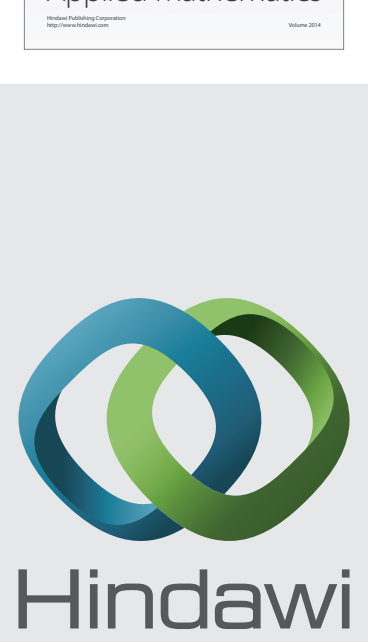

Submit your manuscripts at http://www.hindawi.com
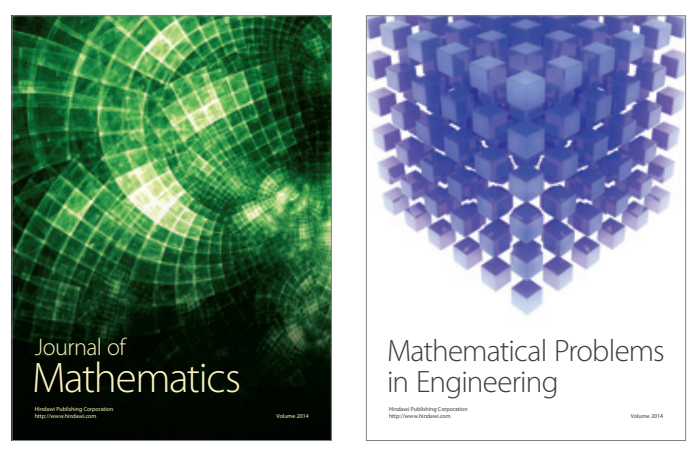

Mathematical Problems in Engineering
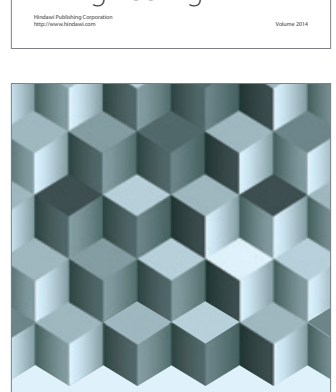

Journal of

Function Spaces
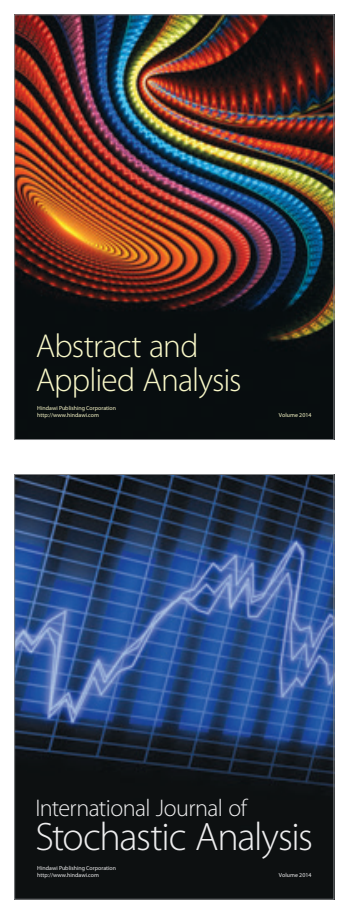

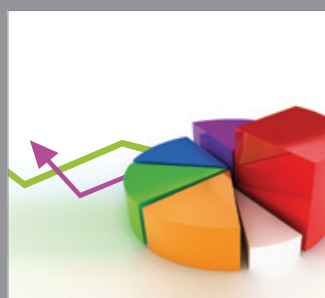

ournal of

Probability and Statistics

Promensencen
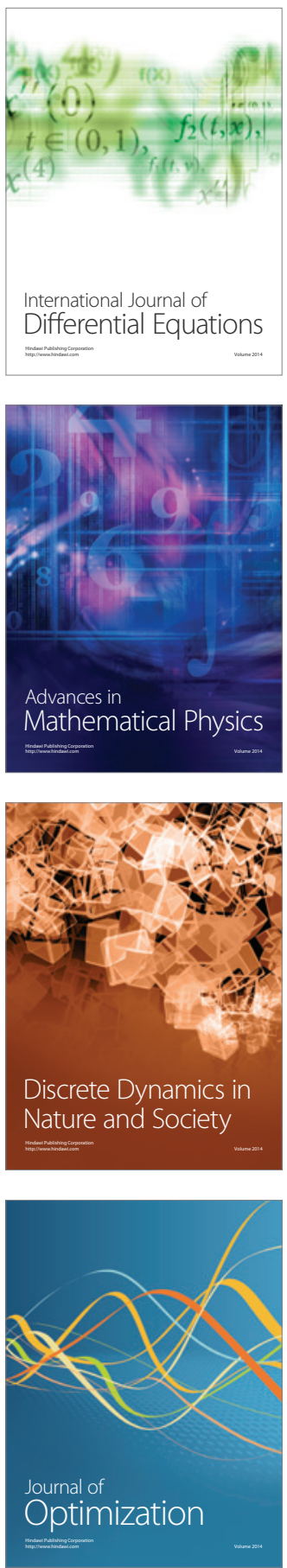